\title{
A GLRT Approach for Detecting Correlated Signals in White Noise in Two MIMO Channels
}

\author{
I. Santamaria, J. Via \\ Dept. Ingeniería de Comunicaciones \\ Universidad de Cantabria \\ Plaza de la Ciencia, Santander, 39005, Spain \\ \{i.santamaria, javier.via\}@unican.es
}

\author{
L. L. Scharf \\ Depts Mathematics, Statistics \\ Colorado State University \\ Fort Collins, CO, 80523, USA \\ scharf@engr.colostate.edu
}

\author{
Y. Wang \\ Dept Mathematics and Statistics \\ Washington State University \\ Pullman, WA, USA, 99163 \\ yuan.wang.stat@gmail.com
}

\begin{abstract}
In this work, we consider a second-order detection problem where rank- $p$ signals are structured by an unknown, but common, $p$-dimensional random vector and then received through unknown $M \times p$ matrices at each of two $M$-element arrays. The noises in each channel are independent with identical variances. We derive generalized likelihood ratio (GLR) tests for this problem when the noise variance is either known or unknown. The resulting detection problems may be phrased as two-channel factor analysis problems.

Index Terms-Passive detection, MIMO channels, passive
\end{abstract} radar, generalized likelihood ratio.

\section{INTRODUCTION}

This paper is motivated by a problem in passive radar, where the problem is to determine if there are complex demodulations and synchronizations in several surveillance antennas (or antenna arrays) that bring signals in the surveillance antennas into coherence with signals in the reference antennas [1]. The conventional approach for passive detection uses the cross-correlation (CC) between the data received in the reference and surveillance channels as the test statistic [2]. However, the noise in the reference signal renders the CC detection scheme suboptimal, especially in multiple-input multiple-output (MIMO) scenarios for which the inherent subspace structure of the received signals can be exploited [3], [4].

Detection of a subspace signal of dimension-one with a single array of sensors under white noise of unknown level has been addressed in [5], [6] and extensions to diagonal noise covariance matrices and dimension- $p$ signals can be found in [7], and [8], [9], respectively. Other variants of this problem, motivated by cognitive radio and multi-static radio applications, have been considered in [10]-[15]. References [12], [13] are noteworthy for their use of a noninformative prior, in this case the Haar measure on the space of dimension- $p$ subspaces, followed by integration for a marginal measurement density. Different from these detection problems, which except for [13] are solved with a single array of sensors at the surveillance channel (for radar applications) or at the secondary user (for cognitive radio applications), the model considered in this paper is solved with the assistance of an additional multiantenna reference channel which acquires a noisy and distorted version of the transmitted signal.
This problem of detecting correlated subspace signals in two MIMO channels may be cast as a problem in factor analysis, where there are constraints on the factor loadings and the factors. In this paper, we derive generalized likelihood ratio (GLR) tests when the Gaussian noises at all antennas are assumed to be independent and identically distributed (i.i.d.), and the variance is either known or unknown. This idealized model may apply when reference and surveillance channels are formed from separate beamsteers in a single array of antenna elements. The maximum likelihood (ML) estimates of channels or factor loadings are determined by using a noise-whitening trick, [16], [17] to construct a noise-whitened version of the sample covariance matrix, and then using a result from [17], [18] to optimize over factor loadings.

Notation: The superscripts $(\cdot)^{T}$ and $(\cdot)^{H}$ denote transpose and Hermitian, respectively. The determinant and trace of a matrix $\mathbf{A}$ will be denoted, respectively, as $\operatorname{det}(\mathbf{A})$ and $\operatorname{tr}(\mathbf{A})$. $\mathbf{I}_{M}$ is the identity matrix of dimensions $M \times M$, and $\mathbf{0}$ denotes the zero matrix of appropriate dimensions. The expectation operator will be denoted by $E[\cdot]$, and $\mathbf{x} \sim \mathcal{C N}_{M}(\mathbf{0}, \mathbf{R})$ indicates that $\mathbf{x}$ is an $M$-dimensional complex circular Gaussian random vector of zero mean and covariance $\mathbf{R}$.

\section{Problem Formulation}

\section{A. Signal Model}

We consider the problem of target detection in a passive network consisting of a reference channel and a surveillance channel, both equipped with $M$ antennas. Our two-channel measurement model is

$$
\left[\begin{array}{c}
\mathbf{x}_{s}[n] \\
\mathbf{x}_{r}[n]
\end{array}\right]=\left[\begin{array}{c}
\theta \mathbf{H}_{s} \\
\mathbf{H}_{r}
\end{array}\right] \mathbf{s}[n]+\left[\begin{array}{c}
\mathbf{v}_{s}[n] \\
\mathbf{v}_{r}[n]
\end{array}\right] ; \quad n=1,2, \ldots, N
$$

where $\mathbf{x}_{s}[n] \in \mathbb{C}^{M}$ and $\mathbf{x}_{r}[n] \in \mathbb{C}^{M}$ are the surveillance and reference measurements; $\mathbf{s}[n] \in \mathbb{C}^{p}$ contains the signal transmitted by $p$ opportunistic illuminators, $\mathbf{H}_{s} \in \mathbb{C}^{M \times p}$ and $\mathbf{H}_{r} \in \mathbb{C}^{M \times p}$ represent the $M \times p$ channels from the transmitter(s) to the surveillance and reference multiantenna receivers, respectively. The parameter $\theta \in\{0,1\}$ determines whether or not there is a signal $\mathbf{H}_{s} \mathbf{s}[n]$ in the surveillance channel.

We treat the symbol sequence as a sequence of uncorrelated, circular, Gaussian random vectors with unknown covariance 
$E\left[\mathbf{s}[n] \mathbf{s}[m]^{H}\right]=\operatorname{diag}\left[\beta_{1}^{2}, \ldots, \beta_{p}^{2}\right] \delta[n-m]$. The factor loadings $\mathbf{H}_{s}$ and $\mathbf{H}_{r}$ are unknown, to be identified in a maximum likelihood procedure. Without loss of generality, the symbol variances $\beta_{i}^{2}$ may be absorbed into these factor loadings. The vectors $\mathbf{v}_{s}[n]$ and $\mathbf{v}_{r}[n]$ model the additive noise. For notational convenience, the signal, noise, and channel vectors can be stacked as $\mathbf{x}[n]=\left[\mathbf{x}_{s}[n]^{T}, \mathbf{x}_{r}[n]^{T}\right]^{T}, \mathbf{v}[n]=$ $\left[\mathbf{v}_{s}[n]^{T}, \mathbf{v}_{r}[n]^{T}\right]^{T}$ and $\mathbf{H}=\left[\mathbf{H}_{s}^{T}, \mathbf{H}_{r}^{T}\right]^{T}$, respectively.

The covariance model for the signal component of Eq. (1) is

$E\left[\begin{array}{c}\theta \mathbf{H}_{s} \\ \mathbf{H}_{r}\end{array}\right] \mathbf{s}[n] \mathbf{s}^{H}[n]\left[\begin{array}{ll}\theta \mathbf{H}_{s}^{H} & \mathbf{H}_{r}^{H}\end{array}\right]=\left[\begin{array}{cc}\theta^{2} \mathbf{H}_{s} \mathbf{H}_{s}^{H} & \theta \mathbf{H}_{s} \mathbf{H}_{r}^{H} \\ \theta \mathbf{H}_{r} \mathbf{H}_{s}^{H} & \mathbf{H}_{r} \mathbf{H}_{r}^{H}\end{array}\right]$.

The additive noise is assumed to be temporally white, zeromean Gaussian distributed, and uncorrelated between the surveillance and reference channels. The noise covariance matrix can then be written as

$$
E\left[\mathbf{v}[n] \mathbf{v}[m]^{H}\right]=\mathbf{\Sigma}=\left[\begin{array}{cc}
\boldsymbol{\Sigma}_{s s} & \mathbf{0} \\
\mathbf{0} & \boldsymbol{\Sigma}_{r r}
\end{array}\right] \delta[n-m] \in \mathcal{E}
$$

where $\mathcal{E}$ is a set of structured covariances. Four different structuring sets are plausible models for this application:

- Model 1. Independent and identically distributed (i.i.d) noises with identical variance at both channels: $\boldsymbol{\Sigma}_{s s}=$ $\boldsymbol{\Sigma}_{r r}=\sigma^{2} \mathbf{I}_{M}$.

- Model 2. White noises, but with different variances at the surveillance and reference channels: $\boldsymbol{\Sigma}_{s s}=$ $\sigma_{s}^{2} \mathbf{I}_{M}, \boldsymbol{\Sigma}_{r r}=\sigma_{r}^{2} \mathbf{I}_{M}$.

- Model 3. Uncorrelated noises across antennas, thus yielding diagonal noise covariance matrices, $\boldsymbol{\Sigma}_{s s}$ and $\boldsymbol{\Sigma}_{r r}$, with unknown elements along its diagonal.

- Model 4. Noises with arbitrary spatial correlation: $\boldsymbol{\Sigma}_{s s}$ and $\boldsymbol{\Sigma}_{r r}$ are arbitrary full-rank positive definite (psd) matrices.

Model 4 was considered in our previous works [3] and [19], for rank-one and rank- $p$ signal models, respectively. The rank$p$ detector for Model 4 was also reported in [20] for a different problem. The Models 2 and 3 have recently been discussed in [21]. In this work, we focus on Model 1 and derive GLR tests when the noise variance is either known or unknown.

\section{B. Detection problem}

The passive radar detection problem is to test the hypothesis that the surveillance channel contains no signal, versus the alternative that it does:

$$
\begin{aligned}
& \mathcal{H}_{0}: \theta=0 \\
& \mathcal{H}_{1}: \theta=1
\end{aligned}
$$

Denote by $\mathcal{R}_{0}$ and $\mathcal{R}_{1}$ the set of measurement covariance matrices under the null hypothesis and alternative hypothesis for i.i.d. noises (Model 1), respectively. We have

$$
\begin{aligned}
& \mathcal{R}_{0}=\left\{\mathbf{R}: \mathbf{R}=\left[\begin{array}{cc}
\mathbf{0} & \mathbf{0} \\
\mathbf{0} & \mathbf{H}_{r} \mathbf{H}_{r}^{H}
\end{array}\right]+\sigma^{2} \mathbf{I}_{2 M}\right\} \\
& \mathcal{R}_{1}=\left\{\mathbf{R}: \mathbf{R}=\left[\begin{array}{ll}
\mathbf{H}_{s} \mathbf{H}_{s}^{H} & \mathbf{H}_{s} \mathbf{H}_{r}^{H} \\
\mathbf{H}_{r} \mathbf{H}_{s}^{H} & \mathbf{H}_{r} \mathbf{H}_{r}^{H}
\end{array}\right]+\sigma^{2} \mathbf{I}_{2 M}\right\} .
\end{aligned}
$$

This detection problem essentially amounts to testing between two different structures for the composite covariance matrix under the null hypothesis and alternative hypothesis. It may be written as

$$
\begin{array}{lll}
\mathcal{H}_{0}: \mathbf{x}[n] \sim \mathcal{C N}_{2 M}(\mathbf{0}, \mathbf{R}), & & \mathbf{R} \in \mathcal{R}_{0} \\
\mathcal{H}_{1}: \mathbf{x}[n] \sim \mathcal{C N}_{2 M}(\mathbf{0}, \mathbf{R}), & & \mathbf{R} \in \mathcal{R}_{1} .
\end{array}
$$

There are two possible interpretations of this model: (1) it is a one-channel factor model with special constraint on the loadings under $\mathcal{H}_{0}$; or (2) it is a two channel factor model with constraint under $\mathcal{H}_{0}$ and common factors in the two channels.

These second-order models for the measurements, where the signal modulates the covariance, so to speak, is the model that results when the conditional density for the measurements is marginalized over the distribution of the Gaussian random vectors $\mathbf{s}[n]$. It is important to distinguish this second-order model from first-order models that treat the $\mathbf{s}[n]$ as unknown random vectors to be estimated from the measurements. In the second-order model, there are only the $2 M p$ parameters of $\mathbf{H}$ to be estimated (under the alternative) from the $2 M N$ measurements, resulting in $N / p$ measurements per parameter. In the first-order model there are $2 M p+p N$ parameters to be estimated from $2 M N$ measurements, resulting in $2 M N /(2 M p+p N)$ measurements per parameter. This is a reduction in the number of measurements per parameter by the factor $(1+N / 2 M)$. For $p=1, M=5$, and $N=100$, this factor is 11 . This simple reasoning will help to explain why generalized likelihood reasoning is more effective in secondorder models, when they apply, than is first-order reasoning. And it shows that the second-order model is based on a marginalization over the Gaussian distribution of random $\mathbf{s}[n]$.

\section{The Generalized Likelihood Ratio}

Let us now consider $N$ consecutive array snapshots under a model with generic covariance matrix $\mathbf{R}$

$$
[\mathbf{x}[1] \quad \ldots \quad \mathbf{x}[N]]=\mathbf{X} \in \mathcal{C}^{2 M \times N},
$$

which are i.i.d. realizations of $\mathbf{x}[n] \sim \mathcal{C N}_{2 M}(\mathbf{0}, \mathbf{R})$. As there are unknown parameters under both hypotheses, the NeymanPearson detector is not implementable for this composite test. Therefore, we adopt a Generalized Likelihood Ratio Test (GLRT), which usually results in simple detectors with good performance [17]. The likelihood may be written as

$$
f(\mathbf{X} ; \mathbf{R})=\frac{1}{\pi^{2 M N} \operatorname{det}(\mathbf{R})^{N}} \exp \left\{-N \operatorname{tr}\left(\mathbf{S R}^{-1}\right)\right\},
$$

where $\mathbf{S}=\frac{1}{N} \mathbf{X X}^{H}$ is the sample covariance matrix, partitioned as

$$
\mathbf{S}=\left[\begin{array}{ll}
\mathbf{S}_{s s} & \mathbf{S}_{s r} \\
\mathbf{S}_{s r}^{H} & \mathbf{S}_{r r}
\end{array}\right]
$$

Here $\mathbf{S}_{s s}$ is the sample covariance matrix of the surveillance channel and the other blocks are defined similarly. The generalized likelihood ratio (GLR) is

$$
\Gamma=\frac{f\left(\mathbf{X} ; \hat{\mathbf{R}}_{1}\right)}{f\left(\mathbf{X} ; \hat{\mathbf{R}}_{0}\right)}
$$


where $\hat{\mathbf{R}}_{0}$ and $\hat{\mathbf{R}}_{1}$ are, respectively, the Maximum Likelihood (ML) estimates of the covariance matrix under $\mathcal{H}_{0}$ and $\mathcal{H}_{1}$. They maximize the log-likelihood function

$$
\mathcal{L}(\mathbf{R})=\log \operatorname{det}\left(\mathbf{S R}^{-1}\right)-\operatorname{tr}\left(\mathbf{S R}^{-1}\right) .
$$

\section{ML estimation}

The following theorem is a variation on a result of [18], using a technique of [16] and [17]. It illuminates the problem of estimating the covariance $\mathbf{R}$.

Theorem 1. For a given noise covariance $\mathbf{\Sigma}$, we define the noise-whitened sample covariance matrix

$$
\tilde{\mathbf{S}}=\boldsymbol{\Sigma}^{-1 / 2} \mathbf{S} \boldsymbol{\Sigma}^{-1 / 2}=\left[\begin{array}{ll}
\tilde{\mathbf{S}}_{s s} & \tilde{\mathbf{S}}_{s r} \\
\tilde{\mathbf{S}}_{s r}^{H} & \tilde{\mathbf{S}}_{r r}
\end{array}\right]
$$

with eigenvalue decomposition $\tilde{\mathbf{S}}=\tilde{\mathbf{W}} \tilde{\mathbf{\Lambda}} \tilde{\mathbf{W}}^{H}$, and $\tilde{\mathbf{\Lambda}}=$ $\operatorname{diag}\left(\tilde{\lambda}_{1} \geq \tilde{\lambda}_{2} \geq \ldots \geq \tilde{\lambda}_{2 M}\right) ; \quad \tilde{\mathbf{S}}_{r r}=\tilde{\mathbf{W}}_{r r} \tilde{\mathbf{\Lambda}}_{r r} \tilde{\mathbf{W}}_{r r}^{H}$, and $\tilde{\boldsymbol{\Lambda}}_{r r}=\operatorname{diag}\left(\tilde{\lambda}_{r r, 1} \geq \tilde{\lambda}_{r r, 2} \geq \ldots \geq \tilde{\lambda}_{r r, M}\right)$. Then, under $\mathcal{H}_{1}$, the value of $\mathbf{H H}^{H}$ that maximizes the likelihood (12) is

$$
\mathbf{H H}^{H}=\boldsymbol{\Sigma}^{1 / 2} \tilde{\mathbf{W}} \tilde{\mathbf{D}} \tilde{\mathbf{W}}^{H} \boldsymbol{\Sigma}^{1 / 2}
$$

with $\tilde{\mathbf{D}}=\operatorname{diag}\left(d_{1} \geq d_{2} \geq d_{p} \geq 0 \ldots 0\right)$ and $d_{i}=\max \left(\tilde{\lambda}_{i}-\right.$ $1,0)$. Under $\mathcal{H}_{0}, \theta \equiv 0$, the value of $\mathbf{H}_{r} \mathbf{H}_{r}^{H}$ that maximizes the likelihood (12) is

$$
\mathbf{H}_{r} \mathbf{H}_{r}^{H}=\boldsymbol{\Sigma}_{r r}^{1 / 2} \tilde{\mathbf{W}}_{r r} \tilde{\mathbf{D}}_{r r} \tilde{\mathbf{W}}_{r r}^{H} \boldsymbol{\Sigma}_{r r}^{1 / 2}
$$

with $\tilde{\mathbf{D}}_{r r}=\operatorname{diag}\left(d_{r r, 1} \geq d_{r r, 2} \geq d_{r r, p} \geq 0 \ldots 0\right)$ and $d_{r r, i}=$ $\max \left(\tilde{\lambda}_{r r, i}-1,0\right)$.

Proof. The proof for $\mathcal{H}_{1}$ is identical to Theorem 9.4.1 in [17]. The proof for $\mathcal{H}_{0}$ is straightforward after we rewrite the loglikelihood function using the blockwise decomposition in (13) and use the fact that the noise covariance $\boldsymbol{\Sigma}$ is block diagonal.

For a given $\boldsymbol{\Sigma}$, this theorem gives the value of $\mathbf{H H}^{H}$ that maximizes the log-likelihood function with respect to $\mathbf{R}=\mathbf{H H}^{H}+\boldsymbol{\Sigma}$. Thus, we have the solution $\mathbf{R}=$ $\boldsymbol{\Sigma}^{1 / 2} \tilde{\mathbf{W}} \tilde{\mathbf{D}} \tilde{\mathbf{W}}^{H} \boldsymbol{\Sigma}^{1 / 2}+\boldsymbol{\Sigma}$. Straightforward calculation shows that

$$
\operatorname{det}\left(\mathbf{R S}^{-1}\right)=\prod_{i=1}^{p} \min \left(\tilde{\lambda}_{i}, 1\right) \prod_{j=p+1}^{2 M} \tilde{\lambda}_{j}
$$

and

$$
\operatorname{tr}\left(\mathbf{R S}^{-1}\right)=\sum_{i=1}^{p} \min \left(\tilde{\lambda}_{i}, 1\right)+\sum_{j=p+1}^{2 M} \tilde{\lambda}_{j}
$$

\section{GLRTS}

In this section we present closed-form GLRTs for the i.i.d. noise model when the noise variance is unknown or known. In both cases, the model order $p$ is assumed known and the channel gains $\mathbf{H}_{s}$ and $\mathbf{H}_{r}$ are unknown.

\section{A. GLR detector for unknown $\sigma^{2}$}

Suppose the sample covariance matrices have these eigen decompositions: $\mathbf{S}=\mathbf{W} \boldsymbol{\Lambda} \mathbf{W}^{H}, \mathbf{S}_{s s}=\mathbf{W}_{s s} \boldsymbol{\Lambda}_{s s} \mathbf{W}_{s s}^{H}$ and $\mathbf{S}_{r r}=\mathbf{W}_{r r} \boldsymbol{\Lambda}_{r r} \mathbf{W}_{r r}^{H}$. Under noise model $1^{1}$, the ML solution for $\sigma^{2}$ under the alternative hypothesis is obtained by realizing that $(2 M-p)^{-1} \sum_{p+1}^{2 M} \tilde{\lambda}_{i}=(2 M-p)^{-1} \sum_{p+1}^{2 M} \sigma^{-2} \lambda_{i}$, which returns

$$
\hat{\sigma}_{1}^{2}=\frac{1}{2 M-p} \sum_{i=p+1}^{2 M} \lambda_{i} .
$$

Therefore, the ML estimate of the covariance matrix under the alternative is

$$
\hat{\mathbf{R}}_{1}=\mathbf{W D W} \mathbf{W}^{H}+\hat{\sigma}_{1}^{2} \mathbf{I}_{2 M},
$$

where $\mathbf{D}=\operatorname{diag}\left(d_{1}, \ldots, d_{p}, 0, \ldots, 0\right)$ is an $2 M \times 2 M$ diagonal matrix with $d_{i}=\lambda_{i}-\hat{\sigma}_{1}^{2}$.

The ML estimate of the covariance matrix under the null is

$$
\hat{\mathbf{R}}_{0}=\left[\begin{array}{cc}
\mathbf{0} & \mathbf{0} \\
\mathbf{0} & \hat{\mathbf{H}}_{r} \hat{\mathbf{H}}_{r}^{H}
\end{array}\right]+\hat{\sigma}_{0}^{2} \mathbf{I}_{2 M},
$$

where

$$
\hat{\sigma}_{0}^{2}=\frac{1}{2 M-p}\left(\sum_{i=1}^{M} \lambda_{s s, i}+\sum_{i=p+1}^{M} \lambda_{r r, i}\right),
$$

$\hat{\mathbf{H}}_{r} \hat{\mathbf{H}}_{r}^{H}=\mathbf{W}_{r r} \mathbf{D}_{0} \mathbf{W}_{r r}^{H}, \mathbf{D}_{0}=\operatorname{diag}\left(d_{1}, \ldots, d_{p}, 0, \ldots, 0\right)$ is an $M \times M$ diagonal matrix, and $d_{i}=\max \left(\lambda_{r r, i}-\hat{\sigma}_{0}^{2}, 0\right)$.

Plugging (17) - (18) into (11), the GLRT under noise model 1 when the noise variance is unknown is given by

$$
\Gamma_{1}=\frac{\left(\prod_{i=1}^{p_{r}} \lambda_{r r, i}\right)\left(\hat{\sigma}_{0}^{2}\right)^{2 M-p_{r}}}{\left(\prod_{i=1}^{p} \lambda_{i}\right)\left(\hat{\sigma}_{1}^{2}\right)^{2 M-p}} \underset{H_{0}}{\gtrless} \eta .
$$

where $p_{r}$ is the largest value of $i$ between 1 and $p$ such that $\lambda_{r r, i}>\hat{\sigma}_{0}^{2}$.

\section{B. GLR detector for known $\sigma^{2}$}

When the noise variance $\sigma^{2}$ is known, the ML estimate of the covariance under the alternative is

$$
\hat{\mathbf{R}}_{1}=\mathbf{W D W} \mathbf{W}^{H}+\sigma^{2} \mathbf{I}_{2 M},
$$

where $\mathbf{D}=\operatorname{diag}\left(\lambda_{1}-\sigma^{2}, \ldots, \lambda_{p_{a}}-\sigma^{2}, 0, \ldots, 0\right)$, and $p_{a}$ is the largest value of $i$ between 1 and $p$ such that $\lambda_{i}>\sigma^{2}$. Likewise, the ML estimate of the covariance matrix under the null when $\sigma^{2}$ is known is

$$
\hat{\mathbf{R}}_{0}=\left[\begin{array}{cc}
\mathbf{0} & \mathbf{0} \\
\mathbf{0} & \mathbf{W}_{r r} \mathbf{D}_{0} \mathbf{W}_{r r}^{H}
\end{array}\right]+\sigma^{2} \mathbf{I}_{2 M},
$$

where $\mathbf{D}_{0}=\operatorname{diag}\left(\lambda_{r r, 1}-\sigma^{2}, \ldots, \lambda_{r r, p_{n}}-\sigma^{2}, 0, \ldots, 0\right)$ with $p_{n}$ being the largest value of $i$ between 1 and $p$ such that $\lambda_{r r, i}>\sigma^{2}$.

Plugging now (21) - (22) into (11), the GLRT under noise model 1 when the variance is known is given by

$$
\Gamma_{2}=\frac{\left(\prod_{i=1}^{p_{n}} \tilde{\lambda}_{r r, i}\right) \exp \left(\sum_{i=1}^{p_{a}} \tilde{\lambda}_{i}\right)}{\left(\prod_{i=1}^{p_{a}} \tilde{\lambda}_{i}\right) \exp \left(\sum_{i=1}^{p_{n}} \tilde{\lambda}_{r r, i}\right)} e^{\left(p_{n}-p_{a}\right)} \underset{H_{0}}{\stackrel{H_{1}}{\gtrless}} \eta,
$$

${ }^{1}$ We focus here on the case where $p \leq 2 M-1$; otherwise the spatial structure of the target plays no role and the GLRT detector is given by the well-known sphericity test [22]. 
with $\tilde{\lambda}_{i}=\lambda_{i} / \sigma^{2}$ and $\tilde{\lambda}_{r r, i}=\lambda_{r r, i} / \sigma^{2}$.

\section{Simulation Results}

In this section we evaluate the performance of the proposed GLR tests for the i.i.d. noise model by means of Monte Carlo simulations. The input signal-to-noise-ratio (SNR) for both the surveillance and reference channels is defined as

$$
\mathrm{SNR}_{j}=10 \log _{10} \frac{\frac{1}{M} \operatorname{tr}\left(\mathbf{H}_{j}^{H} \mathbf{H}_{j}\right)}{\sigma^{2}}, \quad j=\{s, r\} .
$$

The observations under each hypothesis are generated as $\mathbf{x}[n] \sim \mathcal{C N}_{2 M}\left(\mathbf{0}, \mathbf{R}_{i}\right), i=\{0,1\}$, with $\mathbf{R}_{0}$ and $\mathbf{R}_{1}$ having the structure in (5) and (6), respectively. We collect $N$ snapshots and compute the test statistic (20) for unknown $\sigma^{2}$, and (23) for known $\sigma^{2}$.

The first example involves a scenario with $M=6$ antennas, $N=50$ snapshots, $\mathrm{SNR}_{s}=-10 \mathrm{~dB}$ and $\mathrm{SNR}_{r}=-5 \mathrm{~dB}$. Fig. 1 depicts the Receiver Operating Characteristic (ROC) curve for the GLR tests for $p=1$ and $p=3$. The performance for the GLR detectors is better for rank-one signals, since there is more spatial structure to exploit, and there are more measurements per parameter to be estimated. For the same reason, the benefit of knowing $\sigma^{2}$ is only marginal for $p=1$, while it is more significant for $p=3$.

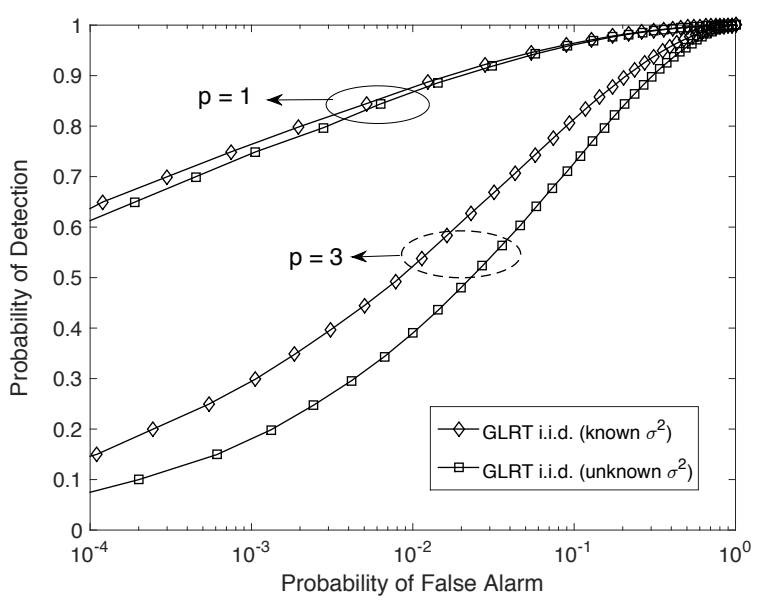

Fig. 1. ROCs for a scenario with $M=6$ antennas, $N=50$ snapshots, $\mathrm{SNR}_{s}=-10 \mathrm{~dB}$ and $\mathrm{SNR}_{r}=-5 \mathrm{~dB}, N / p=50,50 / 3$.

In the second example we consider a scenario with $M=8$ antennas, $\mathrm{SNR}_{s}=\mathrm{SNR}_{r}=-10 \mathrm{~dB}$, and a rank-one signal. Fig. 2 depicts the ROC curves for the GLR tests with $N=20$ and $N=50$ snapshots. As we observe, knowing the true $\sigma^{2}$, in addition to being a model of limited practical importance, does not provide a significant benefit.

In the last example, we study the robustness of the proposed GLR tests under mismatched noise models. We consider a scenario with $M=5$ antennas, a rank-one signal and $N=50$ snapshots. In the detectors, the noises are assumed to have equal variances. But in the experiments, the noises at

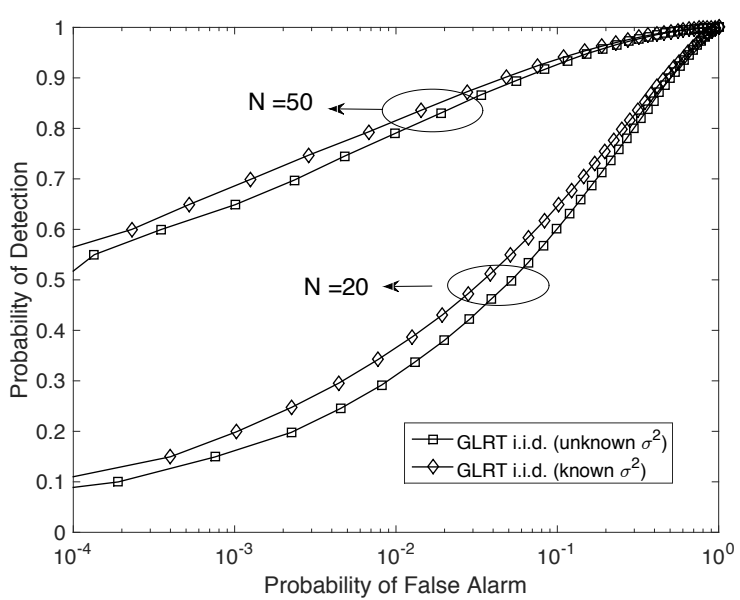

Fig. 2. ROCs for a scenario with $M=8$ antennas, $\mathrm{SNR}_{s}=\mathrm{SNR}_{r}=-10$ $\mathrm{dB}$, and $p=1 ; N / p=20,50$

both arrays are uncorrelated with different variances or have arbitrary spatial correlation, which corresponds respectively to Models 3 and 4 as described in Section II. For comparison we include the GLRT under Model 4, which compares the product $\prod_{1}^{p}\left(1-k_{i}^{2}\right)$ to a threshold, where the $k_{i}$ 's are squared canonical coordinates of the two channel sample covariance matrix [19], [20]. For the GLRT with known noise variance we use $\sigma^{2}=\frac{\operatorname{tr}(\boldsymbol{\Sigma})}{2 M}$. Fig. 3 shows the results obtained for different SNR values. Not surprisingly, the GLRT in [19] provides the best results for both noise models (it is matched to Model 4). In general, the GLRT with assumed unknown noise variance is more robust against mismatched noise models than is the GLRT with assumed known noise variance.

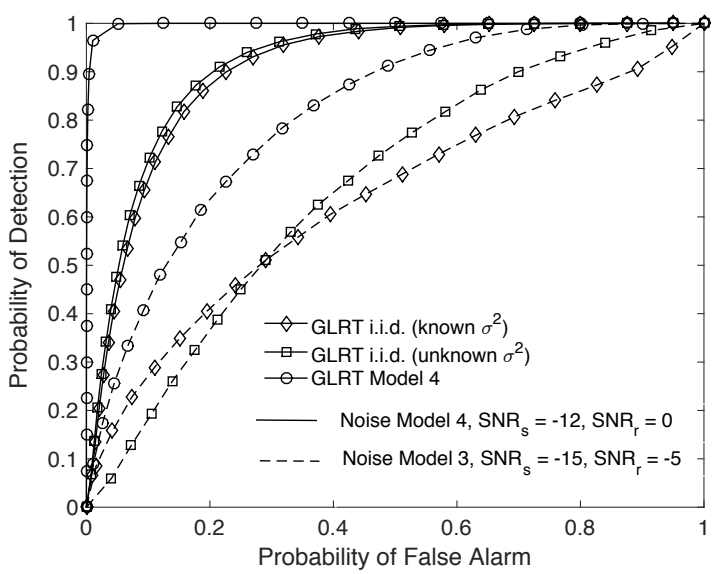

Fig. 3. Performance under mismatched noise models for a scenario with $M=5$ antennas, $p=1$, and $N=50$ snapshots, $N / p=50$. 


\section{CONCLUSION}

In this paper we have addressed a second-order detection problem motivated by passive radar. The problem is to detect a common rank- $p$ signal in two MIMO channels. It turns out that the problem is a problem in factor analysis, where there are constraints on the factor loadings and the factors. We have derived the GLRTs for the case of common noise variances in the two MIMO channels when the noise variance is unknown or known. Numerical simulations indicate that the GLRT for unknown $\sigma^{2}$ is more robust and of more practical interest than the test for known noise variance, which is a model of more limited applicability.

The second-order model we assume in our detectors, with the signal component appearing as a rank- $p$ component in the measurement covariance, amounts to a marginalization over the Gaussian distribution of the symbols transmitted from transmitters of opportunity. This leaves only the channel matrices to be estimated in a GLR theory, or marginalized out in a Bayesian theory. For the examples we have considered, where the number of measurements per parameter is on the order of 50 , the second-order GLR appears to perform satisfactorily.

\section{ACKNOWLEDGMENTS}

The authors wish to acknowledge fruitful discussions of this problem with Douglas Cochran, Stephen Howard, Dan Hack, and Lee Patton. The work of I. Santamaria and J. Via has been supported by the Ministerio de Economía, Industria y Competitividad (MINECO) of Spain, and AEI/FEDER funds of the E.U., under grants TEC2013-47141-C4-R (RACHEL), TEC2016-75067-C4-4-R (CARMEN), and TEC2016-81900REDT (KERMES).

\section{REFERENCES}

[1] D. E. Hack, L. K. Patton, B. Himed, M. A. Saville "Detection in passive MIMO radar networks," IEEE Trans. Signal Process., vol. 62, no. 11, pp. 2999-3012, June 2014

[2] J. Liu, H. Li, B. Himed, "On the performance of the cross-correlation detector for passive radar applications," Signal Process., vol. 113, pp. 32-37, 2015

[3] I. Santamaria, L. L. Scharf, D. Cochran, J. Via, "Passive detection of rank-one signals with a multiantenna reference signal," in European Signal Processing Conference (EUSIPCO), Budapest, Hungary, Sept. 2016.

[4] Y. Wang, L. L. Scharf, I. Santamaria, H. Wang, "Canonical correlations for target detection in a passive radar network," in Asilomar Conference on Signals, Systems and Computers (ASILOMAR), Pacific Grove (CA), USA, November, 2016.

[5] O. Besson, S. Kraut, L. Scharf, "Detection of an unknown rank-one component in white noise," IEEE Trans. Signal Process., vol. 54, no. 7, pp. 2835-2839, July 2006.

[6] O. Besson, L. Scharf, "CFAR matched direction detector," IEEE Trans. Signal Process., vol. 54, no. 7, pp. 2840-2844, July 2006.

[7] D. Hack, L. Patton, B. Himed, "Multichannel detection of an unknown rank-one signal with uncalibrated receivers" IEEE Int. Conf. Acoust., Speech and Signal Proc. (ICASSP), Florence, Italy, 2014

[8] D. Ramirez, G. Vazquez-Vilar, R. Lopez-Valcarce, J. Via, I. Santamaria, "Detection of rank-P signals in cognitive radio networks with uncalibrated multiple antennas," IEEE Trans. Signal Process., vol. 59, no. 8 , pp. 3764 - 3774, Aug. 2011

[9] D. E. Hack, C. W. Rossler, L. K. Patton, "Multichannel detection of an unknown rank-N signal using uncalibrated receivers," IEEE Signal Process. Lett., vol. 21, no. 8, pp. 998-1002, Aug. 2014.
[10] A. Taherpour, M. Nasiri-Kenari, S. Gazor, "Multiple antenna spectrum sensing in cognitive radios," IEEE Trans. Wireless Commun., vol. 9, pp. 814-823, Feb. 2010.

[11] J. Sala-Alvarez, G. Vazquez-Vilar, R. Lopez-Valcarce, "Multiantenna GLR detection of rank-one signals with unknown power spectrum in white noise with unknown spatial correlation," IEEE Trans. Signal Process., vol. 60, no. 6, pp. 3065-3078, June 2012.

[12] S. Sirianunpiboon, S. D. Howard, D. Cochran, "Multiple-channel detection of signals having known rank" IEEE Int. Conf. Acoust., Speech and Signal Proc. (ICASSP), Vancouver, BC, Canada, pp. 6536-6540, 2013.

[13] S. Sirianunpiboon, S. D. Howard, D. Cochran, "Exact Bayesian test for a common rank-one component in white noise" Asilomar Conference on Signals, Systems, and Computers, 2015.

[14] J. K. Tugnait, "On multiple antenna spectrum sensing under noise variance uncertainty and flat fading," IEEE Trans. Signal Process., vol. 60, no. 4, pp. 1823-1832, Apr. 2012

[15] A. Leshem, A.-J. Van der Veen, "Multichannel detection of Gaussian signals with uncalibrated receivers," IEEE Signal Process. Lett., vol. 8, no. 4, pp. 120-122, Apr. 2001.

[16] K. G. Joreskog "Some contributions to maximum likelihood factor analysis," Psychometrika, vol 32, pp. 443-482.

[17] K. V. Mardia, J. T. Kent, and J. M. Bibby, Multivariate Analysis, New York: Academic, 1979, pp. 264-265.

[18] T. W. Anderson, "Asymptotic theory for principal component analysis," Ann. Math. Statist., vol. 34, no. 1, pp. 122-148, 1963.

[19] Y. Wang, I. Santamaria, L.L. Scharf, and H. Wang, "Canonical coordinates for target detection in a passive radar network" 50th Asilomar Conference on Signals, Systems, and Computers, Nov 6-9, 2016.

[20] P. Stoica, K. M. Wong, Q. Wu "On a nonparametric detection method for array signal processing in correlated noise fields," IEEE Trans. Signal Process., vol. 44, no. 4, pp. 1030-1032, Apr. 1996.

[21] I. Santamaria, L. L. Scharf, J. Via, and Y. Wang, "Passive detection of correlated rank-p signals in two MIMO channels," IEEE Trans. Signal Process., submitted Dec 2016.

[22] J. Mauchly "Significance test for sphericity of a normal n-variate distribution," Ann. Math. Statist., vol. 11, 204209, 1940. 